\title{
A Hybrid Double-Focusing Mass Spectrometer-High-Pressure Drift Reaction Cell To Study Thermal Energy Reactions of Mass-Selected Ions
}

\author{
Paul R. Kemper and Michael T. Bowers \\ Department of Chemistry, University of California, Santa Barbara, California, USA
}

\begin{abstract}
A new instrument is described that couples a reverse-geometry mass spectrometer operating at $5 \mathrm{kV}$ to a high-pressure, temperature-variable drift reactor that operates at thermal energies. The first-stage mass spectrometer is a home-built instrument with the same dimensions and ion optics as a V.G. Instruments ZAB-2F. Ions exiting this instrument are decelerated to between 0 and $10 \mathrm{eV}$ and focused on the entrance hole of the drift cell. The drift cell is $4.0 \mathrm{~cm}$ long and $1.52 \mathrm{~cm}$ in diameter and has a uniform drift field provided by stepped voltages applied to eight field guard rings. Pressure in the cell can be varied from 0 to 2 torr, and temperatures from 100 to $500 \mathrm{~K}$. Ions exiting the cell are accelerated, passed through a quadrupole mass filter, and detected by an electron multiplier. A description is given of the ion optics used to focus and decelerate the beam from the first-stage mass spectrometer. Substantial analysis and discussion are given to ion energies in the cell and methods of pressure measurement. Experimental results obtained compare well with data in the literature when available. A new result on mobilities of $\mathrm{Co}^{+}$ions in helium is presented. The data indicate that ions are present in both the ground state and metastable excited electronic state, exhibiting substantially different mobilities. (J Am Soc Mass Spectrom 1990, 1, 197-207)
\end{abstract}

$\mathrm{T}$ The need for mass-selected ions in studies of ion-neutral interactions is obvious, and a number of instruments have been developed to meet this need. They include the selected ion flow tube (SIFT) [1], the combination SIFT and drift tube (SIFTDRIFT) [2], the tandem flowing afterglow [3], the tandem ion-cyclotron resonance spectrometer (TICR) [4], the ion beam experiment of Armentrout and Beauchamp [5] and guided ion beam instrument of Ervin and Armentrout [6], external source Fourier transform ICR $[7,8]$, the dissociative scattering instrument recently developed by Shukla, Futrell, and coworkers [9], the quadrupole/reaction cell/quadrupole instruments of Bohringer and Arnold $[10,11]$ and of Jarrold et al. [12], and numerous multiple quadrupole and tandem mass spectrometry techniques.

The instrument described here uses a doublefocusing reverse-geometry mass spectrometer as its first stage [13]. Such an arrangement allows the use of different ion sources, including a metal ion cluster source, for the first stage. It is also possible to study the reactions of metastable decomposition products and photoexcited ions. The ions from the mass spectrometer are decelerated from $5 \mathrm{keV}$ to $\sim 3 \mathrm{eV}$ and

Address reprint requessts to Michael T. Bowers, Department of Chemistry, University of California, Santa Barbara, CA 93106. are focused onto the entrance orifice of a high-pressure reaction cell. The pressure in the cell can be varied between $\sim 0.8$ and 2.0 torr of buffer gas (usually helium), with $10^{-5}-10^{-2}$ torr of reactant gas. These buffer gas pressures are high enough to ensure complete translational thermalization of the injected ions before the ions encounter a neutral reactant species (discussed below). Ions are drifted through the cell with a small electric field. Ions that exit the cell are analyzed in a quadrupole mass spectrometer. The temperature of the cell can be varied from $100 \mathrm{~K}$ to $500 \mathrm{~K}$.

Applications of the instrument include studies of ion-neutral bimolecular and clustering reactions and ion mobilities. Examples are given below.

\section{The Instrument}

In this section the discussion is limited to the highpressure drift cell and associated ion optics. The details of the home-built double-focusing first stage are very similar to those of the V.G.ZAB-2F instrument that has been previously described [13].

\section{Vacuum}

The vacuum system (Figure 1) consists of a 10 -in.diameter main chamber (containing the deceleration lenses and the reaction cell), which is pumped with 

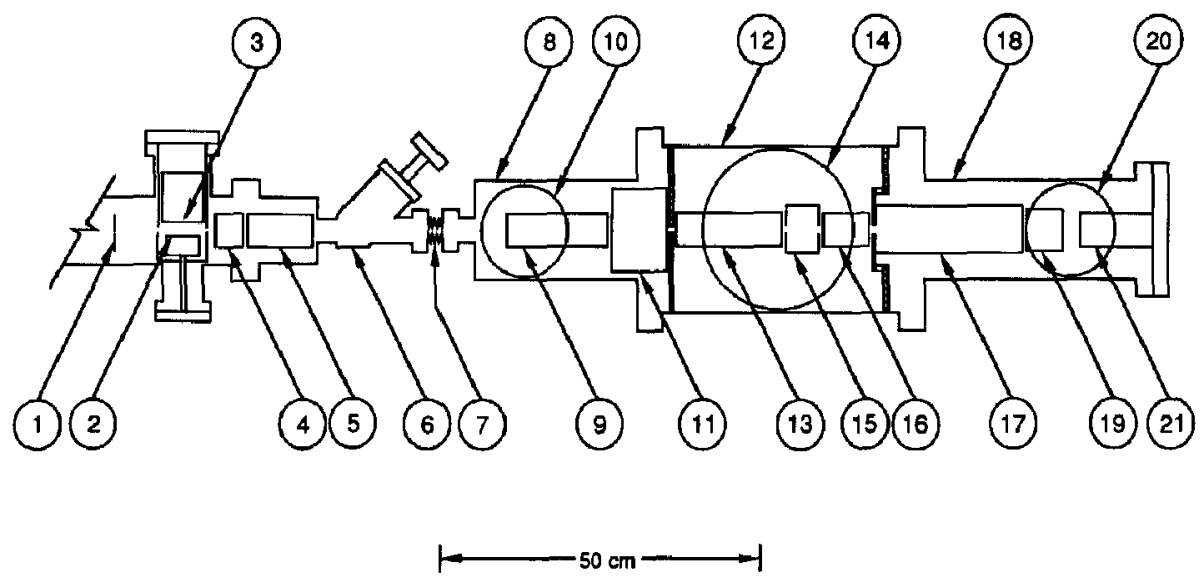

Figure 1. Block diagram showing first-stage mass spectrometer collector region with ion lenses, gas cell, and quadrupole. 1 , Collector slit; 2 , conversion dynode; 3 , electron multiplier; $4, y, z$ steering; 5 , first set of refocusing lenses; 6 , isolation valve; 7 , bellows; 8 , isolation vacuum chamber; 9 , second set of refocusing lenses; 10, 6-in. diffusion pump; 11, $z$ focus and steering lens; 12 , main chamber; 13, deceleration lens; 14,10 -in. diffusion pump; 15 , reaction cell; 16 , quadrupole entrance lens; 17 , quadrupole; 18, quadrupole chamber; 19 , isolation lens; 20, 6-in. diffusion pump; 21 , electron multiplier.

a 10-in. diffusion pump. The quadrupole chamber follows immediately and is pumped with a 6 -in. diffusion pump. Preceding the main chamber is an isolation region that interfaces the first-stage mass spectrometer and the main chamber, also pumped with a 6-in. diffusion pump. The diffusion pumps have a common foreline purmped by two 18-cfm pumps. A separate inlet system pumps the various sample lines.

The pressure isolation between the cell and main chamber changes with cell pressure (discussed below), but at high pressure $p_{\mathrm{CELL}} / p_{\mathrm{MC}} \cong 3000$ with the normal 1-mm cell apertures (maximum cell pressure $\sim 2$ torr). Conductance between the main and quadrupole chambers is via the quadrupole entrance ( $\sim 6 \mathrm{~mm}$ ), and the corresponding isolation is $\sim 100: 1$, for a maximum pressure of approximately $6 \times 10^{-6}$ torr in the quadrupole chamber. The quadrupole shroud was vented for greater pumping. Communication between the main chamber and isolation region is via a $2 \mathrm{~mm} \times 10 \mathrm{~mm}$ slit. The corresponding isolation is $\sim 200: 1$. This stage of differential pumping is necessary to maintain a low pressure in the ESA and collector regions of the first-stage mass spectrometer. An isolation valve is fitted between the mass spectrometer and the isolation region.

Pressures are measured with ionization gauges (one head in each chamber) and a 10-torr capacitance manometer connected directly to the cell.

The cell and all lenses are supported on four 1/4-in. alumina rods that extend through the main chamber and into both the quadrupole and isolation chambers. This ensures that the cell, lenses, slits, and quadrupole are aligned. The cell is easily removed and replaced through a 6 -in. flange atop the main chamber. All heating/cooling and gas connections are made through this flange.

\section{Cell}

General. A cross section of the reaction cell is shown in Figure 2. The cell consists of a cylindrical copper shroud that contains the heating/cooling passages, two end caps (also copper), and a series of eight field guard rings that produce the uniform drift field ${ }^{1}$ The drift voltage is applied to the exit cap, which is electrically isolated. A resistor chain provides the proper voltages to the rings. The drift volume is $4.0 \mathrm{~cm}$ long by $1.52 \mathrm{~cm}$ in diameter. The entrance and exit plates are molybdenum $0.012 \mathrm{~cm}$ thick. The entrance and exit orifices are $0.1 \mathrm{~cm}$. This arrangement allows a maximum cell pressure of $\geq 2$ torr when helium is used as the buffer gas. Separate connections are made to the reactant and helium gas inlets and to the capacitance manometer. These connections must be isolated to $5 \mathrm{kV}$ at pressures from 0 to 2 torr. The cell temperature can be varied from $\sim 80 \mathrm{~K}$ to $600 \mathrm{~K}$. The minimum temperature is reached by flowing liquid $\mathrm{N}_{2}$ through the shroud. Cell temperatures between $150 \mathrm{~K}$ and $300 \mathrm{~K}$ are obtained by varying the flow rate of cold $\mathrm{N}_{2}$ gas through the shroud. The $\mathrm{N}_{2}$ is cooled by flowing it through a coil suspended in liquid $\mathrm{N}_{2}$. Temperature regulation of +2 $\mathrm{K}$ is routine. Cell temperatures between $300 \mathrm{~K}$ and 600 $\mathrm{K}$ are obtained by the variable heating of a constant $\mathrm{N}_{2}$ flow. Here the $\mathrm{N}_{2}$ temperature is measured before it enters the cell and the power supplied to the two 200$W$ in-line heaters used to heat the $\mathrm{N}_{2}$ gas is regulated accordingly. Temperature regulation is about $\pm 1 \mathrm{~K}$. The temperature is measured with a thin-film platinum resistance thermometer (Omega Engineering, Inc., Stamford, CT) suspended in the cell bath gas.

Pressure measurement. Reactant gas pressures are be-

${ }^{1}$ A uniform field can also be produçed by making the cell diameter $\geq 3 \times$ length. In our case the restulting cell was too large. See ref. 12 . 


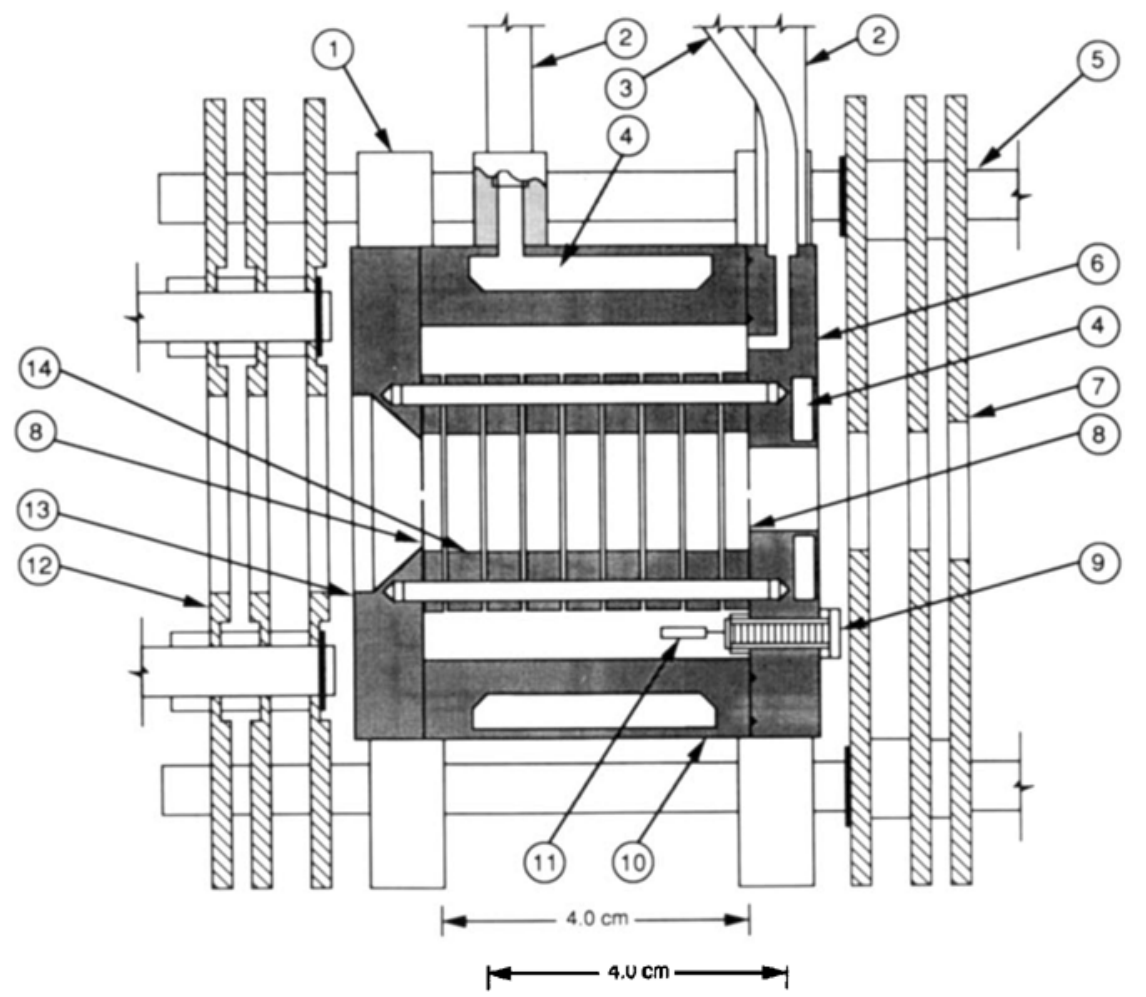

Figure 2. Reaction cell. 1, Cell support bracket; 2, cooling tubes; 3 , gas connection (one of three); 4, cooling passage; 5 , ceramic rod; 6 , exit cap plate; 7 , quadrupole entrance lens; 8 , orifice plate $(0.013-$ $\mathrm{cm}$ thick molybdenum with $0.1-\mathrm{cm}$ hole); 9 , feedthrough for platinum resistance thermometer; 10 , cell body; 11, thin-film platinum thermometer; 12 , deceleration lenses; 13 , entrance cap plate; 14, drift ring.

tween $10^{-5}$ and $10^{-2}$ torr. These cannot be measured directly in the presence of the $\sim 1.5$ torr helium buffer gas pressure. Instead, the main chamber ion gauge is calibrated against cell pressure (as measured on the capacitance manometer) at high pressure and used to set the reactant gas cell pressure without helium. This reactant gas pressure decreases, however, when helium is added, owing to the change in conductance out of the cell. At low cell pressure (effusive flow conditions), the conductance in cubic centimeters per second is $[14,15]$

$$
F_{1}=A\left(\frac{R T}{2 \pi M}\right)^{1 / 2}
$$

where $A$ is the orifice area, $R$ is the gas constant, $T$ is the temperature, and $M$ is the mass. If a high cell pressure $p_{1}$ is present (mean free path $\ll$ orifice diameter), then enthalpy-limited flow occurs from the cell orifice into the main vacuum (at pressure $p_{2}$ ) $[15,16]$, and

$$
F_{\mathrm{h}}=\frac{A r_{\mathrm{c}}}{1-r}{ }^{1 / \gamma}\left[\frac{2 \gamma}{\gamma-1}\left(\frac{R T}{M}\right)\left(1-r_{\mathrm{c}}^{(\gamma-1) / \gamma}\right)\right]^{1 / 2}
$$

where $F_{h}$ is the conductance at high pressure, $\gamma$ is the heat capacity ratio $C_{\mathrm{p}} / C_{\mathrm{v}}, r$ is the pressure ratio $p_{2} / p_{1}$, and $r_{\mathrm{c}}$ is the critical pressure ratio below which no increase in conductance occurs $\left(r_{c}=0.49\right.$ for helium) [15]. A plot of main chamber pressure versus cell pressure shows two linear regions of constant conductance, as predicted by eqs 1 and 2. Incidentally, this result shows that viscous flow from the source does not occur, for conductance at high pressure would then be proportional to pressure [14].

Determining the ratio of low- and high-pressure conductances is critical to determining the reactant gas pressure. This was done in two ways. First, the ratio of the low- and high-pressure slopes of the plot of main chamber pressure versus cell pressure was measured; this ratio equals the ratio of conductances $F_{1} / F_{\mathrm{h}}$. The measured value was $F_{1} / F_{\mathrm{h}}=0.61 \pm 0.04$. Second, a cell pressure of $\sim 10^{-2}$ torr of reactant was set by using the capacitance manometer; helium was added to $\sim 1.5$ torr; the reactant was removed, and the decrease in pressure was noted and compared to the initial value. This ratio was $0.65 \pm 0.05$. These ratios agree well with each other and fairly well with the theoretical value of $F_{1} / F_{\mathrm{h}}=0.55$ obtained from eqs 1 and 2 .

Finally, thermal transpiration must be considered if the cell temperature is varied. If two chambers at different temperatures are connected by a tube through which molecular flow occurs (mean free path $\geq 10 \times$ tube diameter), then $[14,17]$

$$
p_{1} / p_{2}=\left(T_{1} / T_{2}\right)^{1 / 2}
$$


at high pressure (mean free path $\leqslant$ tube diameter/1000), no transpiration occurs and

$$
p_{1} / p_{2}=1.0
$$

Temperature effects at intermediate pressures can be analyzed by the empirical method of Taishacki [17]. Analysis of pressure calibrations (plots of cell pressure versus main chamber pressure) done on our instrument at different temperatures, coupled with the conductance equations, eqs 1 and 2 , indicate that transpiration is well represented by Taishacki's equations. Furthermore, to a good approximation $( \pm 3 \%)$, at low pressure $\left(\leq 4 \times 10^{-3}\right.$ torr in the cell), thermal transpiration occurs between the cell and the manometer (tube $=1.75 \mathrm{~mm}$ diameter) according to eq $3 \mathrm{a}$, whereas at high pressure ( $\geq 0.8$ torr) no transpiration occurs.

Transmission through the cell. If $n_{0}$ ions are created instantaneously at a point $(z=r=0)$ at time $t=0$ and then drift a distance $z$ at average velocity $v_{d}$, the ion current collected through an orifice of radius $r_{\mathrm{c}}$ is given by the ion flux equation $[18,19]$

$$
\begin{aligned}
& \Phi\left(z, t, r_{\mathrm{c}}\right)=\frac{n_{0}}{\sqrt{\pi D_{\mathrm{L}} t}}\left(\frac{1}{4}\right)\left(\frac{z}{t}+v_{\mathrm{d}}\right) \\
& \cdot \exp \left(\frac{-\left(z-v_{\mathrm{d}} t\right)^{2}}{4 D_{\mathrm{L}} t}\right)\left[1-\exp \left(\frac{-r_{\mathrm{c}}^{2}}{4 D_{\mathrm{T}} t}\right)\right]
\end{aligned}
$$

where $D_{\mathrm{L}}$ and $D_{\mathrm{T}}$ are the longitudinal and transverse diffusion coefficients. Equation 4 describes a Gaussian ion packet moving at velocity $v_{\mathrm{d}}$ and spreading longitudinally and radially as it travels through the cell. For a constant current input, the corresponding output current is given by

$$
I_{\text {out }}=\int_{0}^{\infty} \Phi d t
$$

The integration cannot be done analytically. However, the attenuation of the center of the ion packet by the exit orifice can be calculated from the transverse diffusion attenuation term

$$
\text { Attenuation }=1-\exp \left(\frac{-r_{\mathrm{c}}^{2}}{4 D_{\mathrm{T}^{t}}}\right)
$$

The time required for the center of the distribution to traverse the cell $\left(t_{\mathrm{d}}=z / v_{\mathrm{d}}\right)$ ranges from 100 to 500 $\mu \mathrm{s}$ in our experiment. These drift times correspond to transmissions between 0.015 and 0.003 (eq 6), for $\mathrm{Ar}^{+}$ in helium. Under these conditions (symmetric distribution and low transmission), eq 6 provides a very good approximation to the overall transmission of an input current.

Ion drift times. Because changing the neutral gas pressure is a laborious process in our experiment, reactions are studied as a function of time at fixed pressure. Times are changed by varying the drift field across the cell. The effects of this field on ion kinetic energy and reactivity are discussed below.

The ion drift velocity is given by [18]

$$
v_{\mathrm{d}}=K E
$$

where $K$ is the ion mobility $\left[\mathrm{cm}^{2} /(V \cdot s)\right]$ and $E$ is the electric field strength $\left(=V_{\mathrm{d}} / z\right.$, where $V_{\mathrm{d}}$ is the drift potential). The mobility is calculated from the reduced mobility $K_{0}$ by using [18]

$$
K=K_{0}\left(\frac{T}{273}\right)\left(\frac{760}{p_{\mathrm{b}}}\right)
$$

where $p_{\mathrm{b}}$ is the buffer gas pressure in torr. It follows that the time for the center of the ion packet to traverse the cell is given by

$$
t=\frac{z}{v_{\mathrm{d}}}=\text { constant } \times \frac{p_{\mathrm{b}}}{V_{\mathrm{d}}}
$$

To determine reaction times, ions are gated into the cell in 5-10- $\mu$ s-wide packets. No reactant gas is present. The arrival time distribution (ATD) is collected on a multichannel scalar with $10-\mu$ s channel width. The position of the peak maximum can be interpolated to \pm 3 $\mu$ s. A plot of arrival time versus $p_{b} / V_{d}$ has a slope equal to the constant in eq 9 , and the intercept gives the time the ion packet spends in the quadrupole. The plot is very nearly linear except at long times when the peak is distorted by time-dependent transverse diffusion (eq 6). This diffusion causes a small decrease in the observed drift time from that of the Gaussian packet in the cell (1-5\%). This decrease must be taken into account in mobility measurements (discussed below) but not in determining rate coefficients. The average drift time of the observed ion distribution is the desired reaction time, because only those ions that exit the cell (i.e., are observed) are used to determine rate coefficients. Note that this method does not require the mobility to be constant as the drift velocity is varied, again because actual reaction times are measured. This analysis does assume that we can represent a distribution of reaction times by a single, average time. This is true if the extent of reaction increases linearly with time and the distribution is nearly symmetric. Under these conditions, smaller extents of reaction at shorter times are then exactly compensated by larger extents at longer times. Such an approximation is valid only at small extents of reaction when the reaction exponential can be approximated by a one-term expansion (up to $20-30 \%$ reaction). In fact, no noticeable deviations from linearity are observed until $40-50 \%$ of the parent ions have reacted.

An alternative method of determining reaction times, used by Jarrold et al. [12], involves fitting the arrival time distribution to eq 4 by varying $v_{\mathrm{d}}$. Since, un- 
der low field conditions, the ion diffusion coefficients $D_{\mathrm{L}}$ and $D_{\mathrm{T}}$ are equal and are related to the ion mobility by the Einstein relation [18]

$$
D_{\mathrm{L}}=D_{\mathrm{T}}=K \frac{k_{\mathrm{b}} T}{e}
$$

where $k_{\mathrm{b}}$ is the Boltzmann constant and $e$ is the electron charge, all the unknowns in eq 4 can be related to $v_{d}$. This procedure is equivalent to fitting the reduced mobility $\left(K_{0}\right)$. This method was tested in our experiment with $\mathrm{Ar}^{+}$in helium buffer where $K_{0}$ is known. The best fit was obtained with $K_{0} \approx 85 \%$ of the accepted value. The observed discrepancy may be due to increased signal intensity at long times due to a "sweep out" effect. Ions that would normally be lost to the exit plate near the exit orifice are swept out by the escaping helium and are collected. Such a process would produce an apparently lower rate of transverse diffusion and hence a lower mobility. This effect is present in all drift tubes but is accentuated in our experiment, and perhaps more so in Jarrold et al.'s, due to the relatively short drift length.

Ion energy. Two possible sources of excess ion translational energy are present in our experiment: (1) the ion injection energy and (2) the drift field. We need to show that these do not significantly alter the ion's thermal kinetic energy distribution.

Injection energy. Typical ion injection energies are 3-3.5 $\mathrm{eV}$ (the difference between the first-stage ion source and the drift cell potentials). This energy is dissipated in collisions with the helium buffer. From energy and momentum conservation, the average kinetic energy remaining after a hard-sphere collision is

$$
E_{f_{1}}=E_{\mathrm{i}}\left[\frac{M_{\mathrm{i}}^{2}+M_{\mathrm{b}}^{2}}{\left(M_{\mathrm{i}}+M_{\mathrm{b}}\right)^{2}}\right]=E_{\mathrm{i}} \bar{M} ; \quad \text { single collision }
$$

$$
E_{f_{n}}=E_{\mathbf{i}}(\bar{M})^{n} ; \quad n \text { collisions }
$$

where $E_{\mathfrak{f}_{n}}$ and $E_{\mathrm{i}}$ are the final and initial energies, $M_{\mathrm{i}}$ and $M_{\mathrm{b}}$ are the ion and buffer masses, $\bar{M}$ is defined in eq 11 , and $n$ is the number of collisions. With $M_{\mathrm{i}}=100$ $\mathrm{u}, M_{\mathrm{b}}=4 \mathrm{u}$, and $E_{\mathrm{i}}=3 \mathrm{eV}, E_{\mathrm{f}_{1}}=2.78 \mathrm{eV}$ after one collision. After 90 collisions, $E_{f_{90}}=0.003 \mathrm{eV}$ ( $<10 \%$ of thermal at $300 \mathrm{~K}$ ). Two criteria must be met in the experiment: (1) The ion-buffer collision rate must be very much larger than 90 times the ion-reactant-neutral collision rate; (2) the distance traveled in the cell before thermalization must be a small fraction of the total cell length. This second point is often overlooked, but if this is not true, then observed reactions will come from translationally hot ions regardless of the buffer/reactant pressure ratio. The distance traveled before thermalization, $d$, can be calculated from

$$
d=\int_{0}^{t^{\prime}} v d t
$$

where $t^{\prime}$ is the time required for 90 collisions and $v$ is the sum of the ion drift and injection velocities. This assumes a worst case, where all the excess velocity is forward directed. The injection velocity as a function of collision number $n$ is (from eq 11b),

$$
v_{\mathbf{i}_{n}}=\left(\frac{2 E_{\mathrm{i}}}{m_{\mathbf{i}}} \bar{M}^{n}\right)^{1 / 2}=v_{\mathbf{i}}(\bar{M})^{n / 2}
$$

which leads to

$$
d=\int_{0}^{t^{\prime}}\left[v_{\mathrm{d}}+v_{\mathrm{i}} \bar{M}^{[\mathrm{B}] k t / 2}\right] d t
$$

In eq $13, v_{\mathrm{i}}$ is the initial injection velocity, $[B]$ is the buffer density, $k$ is the ion-buffer collision rate coefficient, and the average number of collisions $n=[\mathrm{B}] \mathrm{kt}$. For $p_{\mathrm{He}}=1.5$ torr and $v_{\mathrm{d}}=4 \times 10^{4} \mathrm{~cm} / \mathrm{s}$ (worst case), $d$ is calculated to be $0.4 \mathrm{~cm}$, or $10 \%$ of the cell length.

In our experiment, at low energies it appears that thermalization occurs largely outside the cell entrance owing to collisions with helium escaping the entrance orifice. This suggestion is indicated by the results of pulsing experiments done at various helium pressures, which measured the time between ion injection and collection as a function of ion entrance energy. Above $\sim 5 \mathrm{eV}$ entrance energy, the transit time decreased slightly with increasing energy, indicating increased penetration before thermalization. Below $3-4 \mathrm{eV}$, however, the transit time increased rapidly with decreasing energy, whereas the transmission declined. Below a threshold, no ions were transmitted. This threshold increased with helium pressure (from $\sim 2 \mathrm{eV}$ at 0.9 torr to $\sim 3.7 \mathrm{eV}$ at 1.6 torr). We interpret this threshold as the minimum energy required to just enter the cell against the gas flow, and operate slightly above the threshold. Reactive collisions before the source (at high energy) should be rare, owing to the short time and low reactant neutral gas density. ${ }^{2}$

Another possible source of excess energy is internal excitation of the reactant ions in thermalization collisions. This effect has been investigated in flow-drift experiments [20] and found to be reasonably efficient with argon buffer but generally inefficient with helium. This dependence of ion internal energy on buffer species mass is expected theoretically [21] and is one reason for using helium as the bath gas. It is possible to vibrationally excite ions drifting in a helium

\footnotetext{
${ }^{2}$ One exception to this is charge-stripping reactions that occur between high-energy reactant ions and helium early in the deceleration. These produce high-energy neutrals that can enter the cell without deceleration and ionize the helium bath gas. Ions produced this way account for about $0.1 \%$ of the observed parent ion intensity and are detected by decreasing the injection energy to zero. Helium ions from the ion gauge also find their way into the cell if the gauge is not baffled.
} 
bath at high drift fields [22]. Such excitation (and relaxation) becomes important as the center-of-mass collision energy approaches the vibrational spacing in the ion $[21,22]$. The initial center-of-mass collision energy of an ion of mass $100 \mathrm{u}$ and kinetic energy $\sim 3 \mathrm{eV}$ with helium is $\sim 0.12 \mathrm{eV}$. Consequently, reactant ions with low-frequency vibrational modes could be excited in the deceleration. It does appear, however, that significant amounts of vibrational excitation occur only when $E_{\mathrm{CM}} \gg E_{\mathrm{vib}}[22]$, a situation that can be readily avoided in our experiment.

From the above arguments we conclude that the ion injection energy in our experiments is not likely to be a problem. This is supported by the experiment of Jarrold et al. [12], who examined association reactions between silicon cluster ions and ethylene as a function of injection energy. Even at very high reactant pressures, no effect on the rate coefficients was observed for injection energies up to $20 \mathrm{eV}$ with neon bath gas.

Kinetic energy due to drift field. Drift fields between 0.6 and $2.5 \mathrm{~V} / \mathrm{cm}$ are used in our normal rate coefficient measurements. At $300 \mathrm{~K}$ and 1.5 torr buffer pressure, these correspond to $E / N=1-5$ townsends (Td). Values of $E / N \leq 6 \mathrm{Td}$ are considered low field (i.e., thermal) [18], although this depends on temperature and the ion/buffer mass ratio [18, 21, 22].

As a check for kinetic energy effects, the charge transfer reaction

$$
\mathrm{Ar}^{+}+\mathrm{N}_{2} \rightarrow \mathrm{N}_{2}^{+}+\mathrm{Ar}
$$

was studied as a function of $E / N$ from 1 to $12 \mathrm{Td}$. This reaction is known to have a strong kinetic energy dependence [23] and is discussed below as an example of reactions studied as a function of kinetic energy. Of importance here is that below $E / N \approx 6 \mathrm{Td}$ the rate coefficient was constant with increasing drift field and agreed well with thermal energy flowing afterglow values. Our conclusion is that reactions at $300 \mathrm{~K}$ can be studied up to field strengths of $\sim 6 \mathrm{Td}$ without perturbation due to excess kinetic energy. This maximum field scales with temperature, however, and thus at $100 \mathrm{~K}$ the maximum allowable value of $E / N$ is $\sim 2 \mathrm{Td}$.

\section{Ion Lenses}

Several lens systems have been devised to decelerate the high-energy ions from sector mass spectrometers. Exponential lenses were used by Vestal et al. [24] and by Ervin and Armentrout [6], multistage rectangular tube lenses were used by Futrell and Miller [25] and by Shukla et al. [9]. Ion trajectories in the present lens system were modeled extensively using SIMION. ${ }^{3}$ The lens system (before the cell) consists of two main parts (Figure 3). The first serves to image the mass spectrom-

\footnotetext{
${ }^{3}$ The SIMION program for modeling ion optics was obtained from David A. Dahl, Idaho National Engineering Laboratory, P.O. Box 1625, Idaho Falls, ID 83415, USA.
}

eter collector slit at the entrance slit of the deceleration lens ( $84 \mathrm{~cm}$ away). This rather long distance was dictated by the physical size of the vacuum system. Beginning the deceleration shortly after the collector slit was considered and rejected. This would require either floating the vacuum system or extensively shielding the ions internally. The imaging is done with a pair of cylinder lenses ( $3.8 \mathrm{~cm}$ diameter) in a Heddle configuration [26]; $y$ and $z$ steering lenses were installed near the mass spectrometer collector slit. Both the radial and angular magnifications $\left(M_{\mathrm{r}}\right.$ and $\left.M_{\theta}\right)$ are unity.

The deceleration occurs in two stages. First there is a series of five rectangular tube lenses $(2.0 \mathrm{~cm}$ width, $15.2 \mathrm{~cm}$ height, $0.25 \mathrm{~cm}$ spacing), again in a Heddle configuration. This configuration acts as a zoom lens that decelerates the ions from $5 \mathrm{keV}$ to between 50 and $1000 \mathrm{eV}$ while maintaining a $y$ focus $10.2 \mathrm{~cm}$ from the entrance slit $\left[M_{\mathrm{r}}=M_{\theta}=\left(E_{\mathrm{i}} / E_{\mathrm{f}}\right)^{1 / 4}\right]$. The first element of this lens is at ground potential and is connected as a second $y$ steering lens. The beam is also brought to a $z$ focus $10.2 \mathrm{~cm}$ from the entrance slit by a three-element Einzel lens just before the entrance slit. This lens incorporates $z$ steering as well. Following the zoom lens is a two-element cylindrical deceleration lens $(2.5 \mathrm{~cm}$ diameter) that reduces the remaining ion energy by a factor of 100 for a range of final energies from 0.5 to $10 \mathrm{eV}$. The first element in this lens is common to the last element in the zoom lens, and the transition from rectangular tube to cylinder lens occurs in a field-free section of this element. The second element of the divide by 100 lens is common to the cell entrance plate. At $3 \mathrm{eV}$ final energy, the overall values of $M_{\mathbf{r}}$ and $M_{\theta}$ are 1.4 and 30 , respectively. This large angular magnification is acceptable in our experiment because the ions are focused into a high-pressure cell and quickly thermalized. The ordering of lenses (zoom first) was done for two reasons: (1) to minimize Coulomb expansion of the beam by minimizing the time the ions spend at low kinetic energy and (2) to minimize the defocusing effect of different initial ion kinetic energies.

Ions exiting the reaction cell are accelerated into the quadrupole. The ion energy entering the quadrupole has a strong influence on the transmission and resolution; this energy can be adjusted between 2 and $50 \mathrm{eV}$. The quadrupole and its shroud are thus floated near $+4950 \mathrm{~V}$. Ions exiting the quadrupole pass through another cylinder lens, which shields the quadrupole exit from the vacuum chamber ground plane. This lens also accelerates the ions to about $300 \mathrm{eV}$ and provides some minor focusing of the beam onto the on-axis multiplier.

The rectangular tube and cylinder lenses were built up from plates and spacers stacked on alumina rods. This arrangement has no appreciable effect on the lens field and allows for better pumping of the lens interior. Molybdenum lens elements were used near the cell; stainless steel was used elsewhere.

It is difficult to evaluate the performance of individual parts of the lens/cell/quadrupole system, since the ions are not refocused after entering the cell entrance 

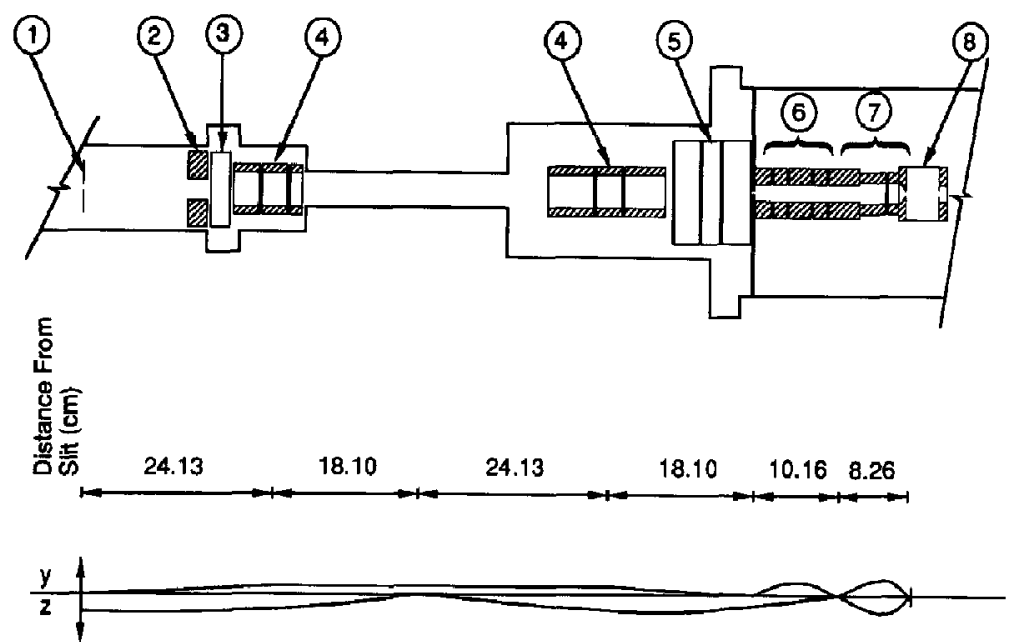

Figure 3. Refocusing and deceleration lenses. 1, Collector slit; 2, y steering; $3, z$ steering; 4 , refocusing lens (3.81-cm-diameter cylinder); $5, z$ focus and steering (rectangular tube lens, $2.54 \mathrm{~cm}$ spacing); 6 , zoom deceleration lens (rectangular tube lens, $2.03 \mathrm{~cm}$ spacing $\times 15.2 \mathrm{~cm}$ tall); 7 , final deceleration (2.54-cm-diameter cylinder); 8 , reaction cell, All lens separations are $0.25 \mathrm{~cm} ; y$ and $z$ ion path displacements are shown schematically and are not to scale. and are collected only after the quadrupole. The overall transmission from the collector of the mass spectrometer to the final collector was measured to be $\sim 4 \times 10^{-4}$ (with $p_{\mathrm{He}}=1.5$ torr and $E=6 \mathrm{~V} / \mathrm{cm}$; mass spectrometer slit widths $=2.0 \mathrm{~mm}$ ). This factor changed only slightly for ion injection energies between 4 and $10 \mathrm{eV}$; below $3 \mathrm{eV}$ it fell sharply (see the discussion of Injection Energy).

\section{Electronics}

Most of the circuitry used is straightforward, but a few points are worth noting. The voltages that follow the cell entrance (drift voltage, quadrupole bias, etc.) track each previous voltage. This allows changes in the drift field or ion entrance energy without readjustment of the quadrupole bias, etc. The quadrupole used (EXTREL Corp., Pittsburgh, PA) could be biased directly to $\pm 5000 \mathrm{~V}$ without modification. Rate data are normally taken as a function of reaction time by changing the drift voltage (discussed above). This drift voltage was placed under computer control by using an opto-isolated 12-bit DAC. The DAC is biased at the cell entrance plate voltage $(\sim 5000 \mathrm{~V})$. This arrangement allows us to measure total rate coefficients by directly observing reactant ion decay as a function of time. This, in turn, eliminates the necessity of scanning over the product ion masses and makes the most efficient use of low reactant ion count rates. Ion detection is done with an on-axis electron multiplier following the quadrupole. The detector is usually operated in pulse counting/single ion detection mode. A multichannel scalar is used to collect the data and is stepped synchronically with the mass or reaction time scan.

\section{Applications}

\section{Rate Coefficients}

As noted above, reactions are studied as a function of time by varying $V_{\mathrm{d}}$ (the drift voltage). Reactant and buffer gas pressures $\left(p_{\mathrm{r}}\right.$ and $p_{\mathrm{b}}$ ) are held constant. It is not possible to measure $p_{\mathrm{r}}$ (or the reactant gas flow) with buffer in the cell. Thus $p_{r}$ is set without buffer and is corrected for the change in cell conductance when buffer is added (see the discussion of Pressure Measurement). The rate coefficient is determined from a plot of $\log$ (extent of reaction) versus time. The extent of reaction can be determined in two ways: (1) multiple quadrupole scans over parent and product ion masses at different drift voltages, or (2) scans of the parent ion intensity versus drift voltage, with and without neutral reactant. The quadrupole scan has two main disadvantages: (1) It makes inefficient use of the reactant ions (important with low count rates), and (2) transmission through the quadrupole may be mass-dependent.

Observation of the parent ion loss with time avoids these problems. In this case, the data are analyzed as follows. Without reactant,

$$
P^{+}(t)=P_{0}^{+} T(t)
$$

where $P^{+}(t)$ is the observed parent intensity, $P_{0}^{+}$is the parent intensity entering the cell, and $T(t)$ is the (unknown) time-dependent transmission probability through the cell and quadrupole. When neutral reactant is added,

$$
P_{\Gamma}^{+}(t)=P_{0}^{+} T(t) e^{-[R] k t}
$$

where $[R]$ is the neutral reactant density and $k$ is the rate coefficient. It follows that

$$
\ln \frac{P_{\mathrm{r}}^{+}(t)}{P^{+}(t)}=-[\mathrm{R}] k t
$$

and the transmission factor is eliminated. Two practical notes regarding this technique:

1. Quadrupole mass peaks often have significant structure, and very small changes in conditions can result in relatively large changes in transmission if 
Table 1. Charge transfer reaction rate coefficients, $\mathrm{Ar}^{+}+\mathrm{M} \stackrel{k}{\rightarrow}$ products

\begin{tabular}{lll}
\hline & \multicolumn{1}{c}{$k, 10^{-10} \mathrm{~cm}^{3} / \mathrm{s}$} \\
\cline { 2 - 3 } $\mathrm{M}$ & This work & Literature \\
\hline $\mathrm{CH}_{4}$ & $9.0 \pm 1$ & $10.0[36], 9.0[37], 9.5[38]$ \\
$\mathrm{O}_{2}$ & $0.37 \pm 0.1$ & $0.46[39], 0.52[40], 0.48[41], 0.39[38]$ \\
$\mathrm{CO}_{2}$ & $4.0 \pm 1$ & $5.5[41], 5.6[42], 7.1[38]$ \\
$\mathrm{N}_{2}$ & $0.1 \pm 0.03$ & $0.11[41], 0.1[34]$ \\
$\mathrm{HCN}$ & $3.5 \pm 1$ & - \\
\hline
\end{tabular}

the quadrupole mass is fixed. This condition leads to poor reproducibility in parent ion loss scans. One solution is to modulate the quadrupole mass by about $\pm 1 / 2 u$ about its center of mass (in effect scanning over the peak). This is done rapidly ( $\sim 1$ $\mathrm{kHz}$ ) so that numerous scans are included in the ion intensity measured at each reaction time.

2. The second point concerns the extent of reaction. Since this method looks at the difference in parent ion loss with and without neutral reactant, this difference should be as large as possible. Thus, the highest possible neutral pressures are used, consistent with an extent of reaction less than $\sim 40 \%$ at the longest times. Also, as discussed above, $E / N$ values must be restricted to avoid nonthermal conditions.

As a check of the instrument's ability to measure thermal energy rate coefficients, the reactions of $\mathrm{Ar}^{+}$ with $\mathrm{CH}_{4}, \mathrm{~N}_{2}, \mathrm{O}_{2}$, and $\mathrm{CO}_{2}$ were studied, along with the reaction with $\mathrm{HCN}$, which has apparently not been reported. The results are summarized in Table 1 . The agreement between present and previous values of rate coefficients is satisfactory, but our results appear to be 10 or $20 \%$ low. This may indicate a systematic error in our experiment (probably in the pressure measurement). In the absence of a systematic error, we estimate the accuracy of the rate coefficient measurements to be $\pm 30 \%$ ( $\pm 15 \%$ pressure measurement, $\pm 10 \%$ reproducibility).

It is also possible to study reactions as a function of kinetic energy by measuring extents of reaction at higher drift fields as in SIFT-DRIFT experiments [2]. A number of authors $[2,18,27]$ have discussed the calculation of ion kinetic energies in these experiments. We give here an outline of the procedure. The kinetic energy of an ion of mass $M_{\mathrm{i}}$ drifting at velocity $v_{\mathrm{d}}$ through a nonreactive bath gas of mass $M_{\mathrm{b}}$ is given by the Wannier expression [27]

$$
\mathrm{KE}_{\text {ion }}=\frac{v_{\mathrm{d}}^{2}}{2}\left(M_{\mathrm{i}}+M_{\mathrm{b}}\right)+\frac{3}{2} k_{\mathrm{b}} T
$$

This is accepted as accurate $( \pm 10 \%)$ at both high and low fields [28]. The center-of-mass (CM) collision energy between the ion and a neutral reactant of mass $M_{\mathrm{r}}$ is

$$
\mathrm{KE}_{\mathrm{CM}}=\frac{1}{2}\left(\frac{M_{\mathrm{r}} M_{\mathrm{i}}}{M_{\mathrm{r}}+M_{\mathrm{i}}}\right)\left(v_{\mathrm{i}}^{2}+v_{\mathrm{r}}^{2}\right)
$$

where

$$
\frac{1}{2} M_{\mathrm{r}} v_{\mathrm{r}}^{2}=\frac{3}{2} k_{\mathrm{b}} T
$$

and

$$
\frac{1}{2} M_{\mathrm{i}} v_{\mathrm{i}}^{2}=\mathrm{KE}_{\mathrm{ton}}
$$

It follows that the center-of-mass reaction energy is

$$
\mathrm{KE}_{\mathrm{CM}}=\frac{M_{\mathrm{r}} v_{\mathrm{d}}^{2}}{2}\left(\frac{M_{\mathrm{i}}+M_{\mathrm{b}}}{M_{\mathrm{i}}+M_{\mathrm{r}}}\right)+\frac{3}{2} k_{\mathrm{b}} T
$$

As an example, our measured dependence of the $\mathrm{Ar}^{+}+\mathrm{N}_{2}$ charge transfer reaction is presented in Figure 4, along with the low-temperature SIFT results of Smith and Adams [29] and the higher energy SIFTDRIFT data of Lindinger et al. [23]. There is good correspondence among the three data sets, well within the combined errors.

\section{Product Distributions}

This application is obvious, but it should be noted that product and parent ions exit from the cell with the same efficiency. This can be seen by considering the

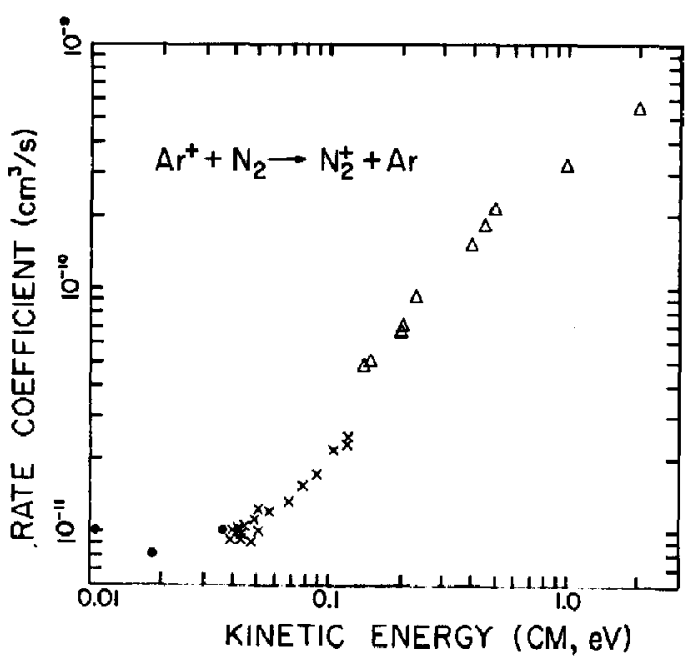

Figure 4. Kinetic energy dependence of $\mathrm{Ar}^{+}+\mathrm{N}_{2}$ charge transfer reaction; variable-temperature SIFT data of Smith and Adams (•) [29], SIFT-DRIFT data of Lindinger et al. $(\Delta)$ [23], present data $(x)$. 
average radial (two-dimensional) displacement of the Gaussian ion packet in the cell [18],

$$
\left(\overline{r^{2}}\right)^{1 / 2}=2\left(D_{\mathrm{T}} t\right)^{1 / 2}
$$

Since the drift time through the cell is

$$
t_{\mathrm{d}}=l / v_{\mathrm{d}}=l / K E
$$

where $l$ is the cell length, it follows from the Einstein relation (eq 10) that at low field

$$
\left.\overline{\left(r^{2}\right.}\right)^{1 / 2}=2 l\left(\frac{k_{\mathrm{b}} T}{e V_{\mathrm{d}}}\right)^{1 / 2}
$$

The radial diffusion, which controls the collection efficiency, is thus independent of the ionic species [18]. Mass-dependent transmission through the quadrupole is minimized by working under high-transmission/lowresolution conditions when possible.

\section{Ion Mobilities}

The use of the present drift cell to measure ion mobilities may be interesting because of the possibility of performing experiments with interesting ionic species such as cluster ions and electronically excited species. Temperature-dependent experiments can also be done. Measurements are restricted to fields $s 20 \mathrm{Td}$ by the apparatus. At these low to medium fields, the ion diffusion coefficients are related to the mobility by a modified Einstein relation derived by Wannier [19, 27]:

$$
\begin{aligned}
& D_{\mathrm{T}}=K \frac{k_{\mathrm{b}} T}{e}+\frac{1}{3} M_{\mathrm{b}} \frac{M_{\mathrm{b}}+M_{\mathrm{i}}}{M_{\mathrm{b}}+1.908 M_{\mathrm{i}}}\left(\frac{E^{2} K^{3}}{e}\right) \\
& D_{\mathrm{L}}=K \frac{k_{\mathrm{b}} T}{e}+\frac{1}{3} M_{\mathrm{b}} \frac{M_{\mathrm{b}}+3.72 M_{\mathrm{i}}}{M_{\mathrm{b}}+1.908 M_{\mathrm{i}}}\left(\frac{E^{2} K^{3}}{e}\right)
\end{aligned}
$$

Thus, measurement of the ion arrival times allows determination of mobilities (eq 7) and diffusion coefficients under these conditions. At higher fields the diffusion coefficients are no longer simply related to the mobility, and their determination requires fitting the observed arrival time distribution to eq 4 [18]. Due to the relatively short drift length in the present cell, these high-field mobilities cannot be determined as accurately as the zero-field mobilities. As noted earlier, the observed time differs from that of the Gaussian packet in the cell $\left(t_{\mathrm{d}}=l / v_{\mathrm{d}}\right)$ because of the transverse diffusion term in the ion flux equation (eq 4). In the near low-field limit where $D_{\mathrm{L}} \cong D_{\mathrm{T}}$, it can be shown that

$$
t_{\mathrm{d}} \cong t_{\mathrm{obs}}+4 D / v_{\mathrm{d}}^{2}
$$

Equation 28 represents a minor correction to $t_{\mathrm{obs}}$ (1-5\%).

As a test of the technique, the mobility of $\mathrm{Ar}^{+}$in helium was determined to be $20 \pm 1.5 \mathrm{~cm}^{2} /(\mathrm{V} \cdot \mathrm{s})$. Reported values range from 19.5 to 27.5 , with the currently accepted value being $\sim 20.4 \mathrm{~cm}^{2} /(\mathrm{V} \cdot \mathrm{s})[30,31]$. A more interesting example involves ground state and excited states of cobalt ions. The ions were formed by low-pressure electron impact on $\mathrm{Co}(\mathrm{CO})_{3} \mathrm{NO}$. The arrival time distribution of the $\mathrm{Co}^{+}$ion is shown in Figure 5. The helium pressure in these preliminary experiments was not high enough to fully separate the peaks. It appears that the two distinct peaks correspond to two electronic states of $\mathrm{Co}^{+}$. Separation of ground and excited electronic state mobilities has been observed with $\mathrm{C}^{+}[32], \mathrm{O}^{+}$[33], and rare gas ions [34]. Evidence for this assignment in our experiment comes from experiments at different ionizing energies. At high energies ( $>30 \mathrm{eV}$ ), the ion with the higher mobility dominates. As the electron energy is reduced, the fraction of slower ion increases, until at $17 \mathrm{eV}$ the fraction of low-mobility ions reaches $90 \%$. A high-resolution mass spectrometer scan of the $\mathrm{m} / \mathrm{z}$ 59 peak showed only one mass, excluding the possibility of an impurity with the same nominal mass. We do not have sufficient data to unambiguously assign specific $\mathrm{Co}^{+}$electronic states to the observed peaks, but they are almost certainly the ${ }^{3} \mathrm{~F}$ ground state and ${ }^{5} \mathrm{~F}$ first excited state. The measured zero-field reduced mobilities were 17.0 and $24.7 \mathrm{~cm}^{2} /(\mathrm{V} \cdot \mathrm{s})( \pm 15 \%)$. It was possible to see that the states did cluster with $\mathrm{C}_{2} \mathrm{H}_{6}$ at different rates, the less mobile $\mathrm{Co}^{+}$ions reacting much faster. This result indicates the feasibility of studying reactions of excited states, and a more detailed account of this mobility and reactivity data will be published elsewhere [35].

\section{Temperature Dependence}

We are examining the three-body association of $\mathrm{Co}^{+}$ with $\mathrm{CH}_{4}$ as a function of temperature, helium pressure, and $\mathrm{CH}_{4} / \mathrm{CD}_{4}$ isotope [43]. Preliminary results are given in Figure 6, which shows the variation of the observed second-order rate coefficient with temperature:

$$
\mathrm{Co}^{+}+\mathrm{CH}_{4} \stackrel{k_{\text {nnd }}^{\text {obs }}}{\rightarrow} \mathrm{Co} \cdot \mathrm{CH}_{4}^{+}
$$

The $\mathrm{Co}^{+}$ions in these experiments were in their ground electronic state. Our results agree well with those of Tonkyn et al. [44].

This reaction almost certainly proceeds according to the mechanism

$$
\mathrm{Co}^{+}+\mathrm{CH}_{4} \underset{k_{\mathrm{b}}}{\stackrel{k_{f}}{\rightleftharpoons}}\left[\mathrm{Co} \cdot \mathrm{CH}_{4}\right]^{+*} \stackrel{\beta k_{3}[\mathrm{He}]}{\longrightarrow} \mathrm{Co} \cdot \mathrm{CH}_{4}^{+}
$$

where $k_{\mathrm{f}}$ is the complex formation rate, $k_{\mathrm{b}}$ is the unimolecular back-dissociation rate, $\beta$ is the stabilization efficiency $(0 \leq \beta \leq 1.0)$, and $k_{\mathrm{s}}$ is the collision rate coefficient for $\left[\mathrm{Co} \cdot \mathrm{CH}_{4}\right]^{+*}$ with helium. It follows from 


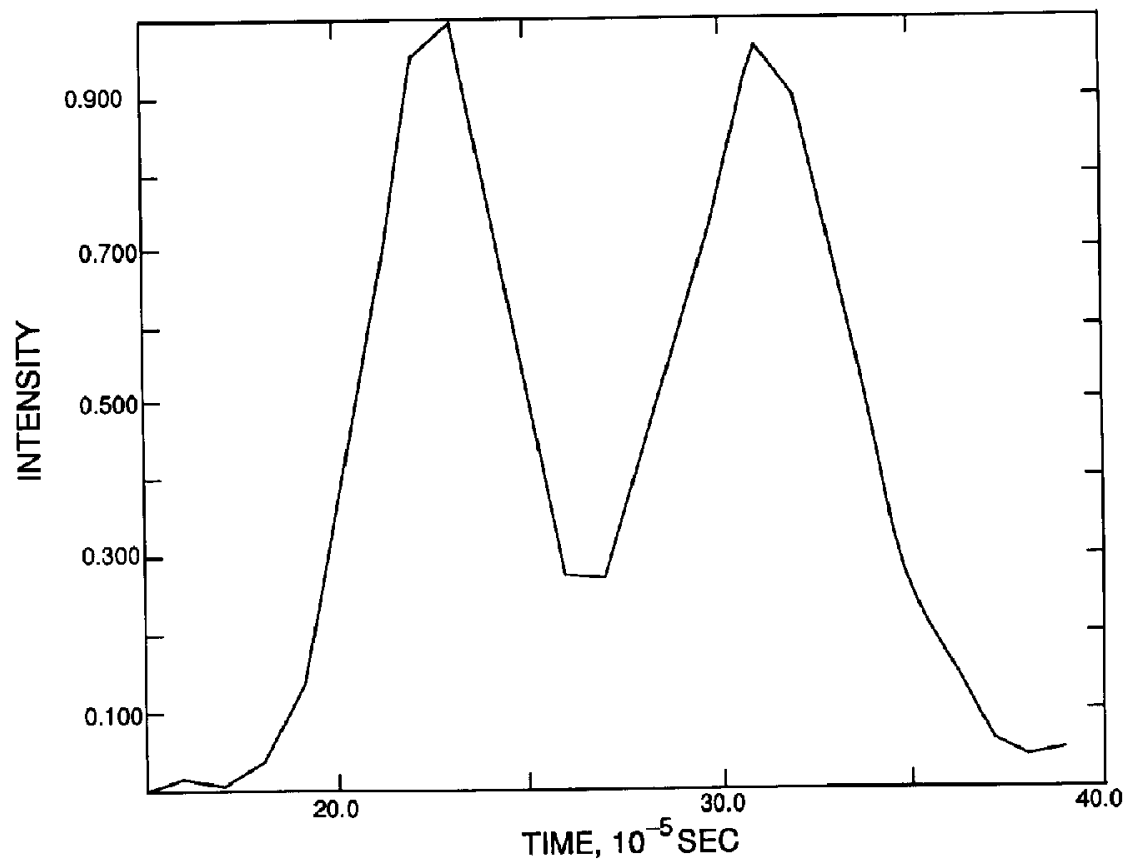

Figure 5. Arrival time distribution of $\mathrm{Co}^{+}$. The $\mathrm{Co}^{+}$was formed by $22-\mathrm{eV}$ electron impact on $\mathrm{Co}(\mathrm{CO})_{3} \mathrm{NO}$ in the source of the first-stage mass spectrometer. The helium pressure in the reaction cell was 1.6 torr, and the drift field $7 \mathrm{~V} / \mathrm{cm}$. The bimodal distribution is assigned to two different electronic states of $\mathrm{Co}^{+}$with different mobilities in helium (see text).

Figure 6. Effect of temperature on $\mathrm{Co}^{+}+\mathrm{CH}_{4}$ clustering rate coefficient. The $[\mathrm{He}]$ is $4 \times 10^{16}$ molecules $/ \mathrm{cm}^{3}$ at all temperatures.

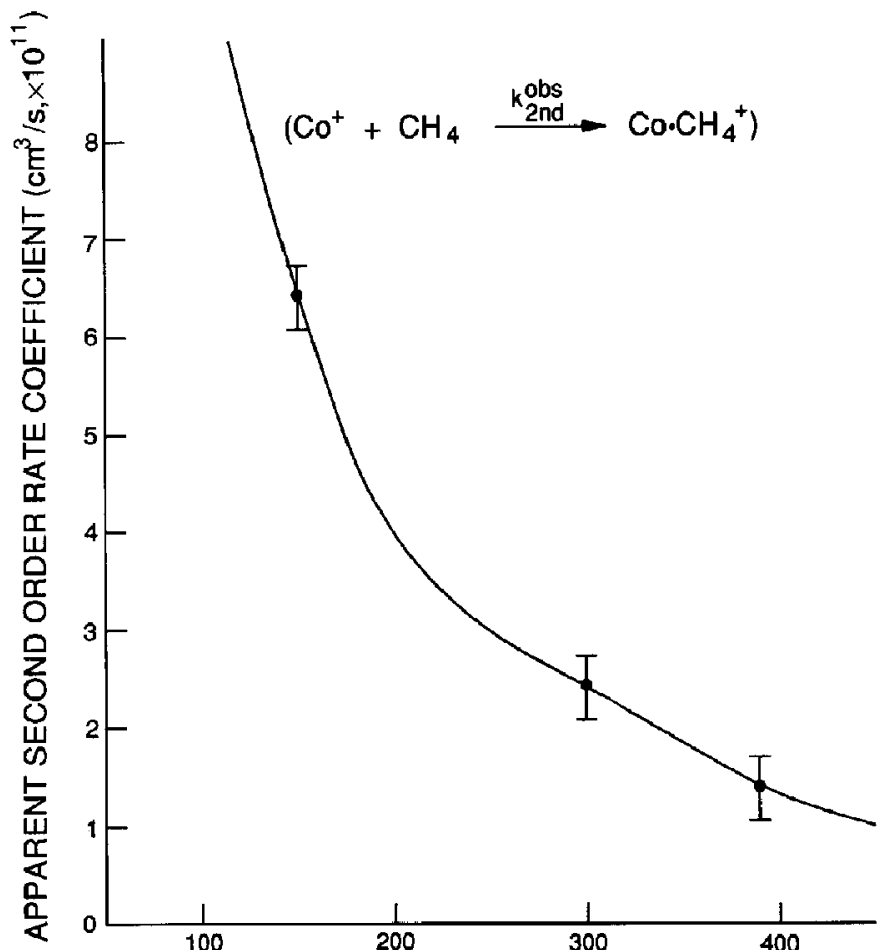

Temperature (K) 
a steady-state assumption that

$$
k_{2 \mathrm{nd}}^{\text {obs }}=\frac{k_{\mathrm{f}} \beta k_{\mathrm{s}}[\mathrm{He}]}{k_{\mathrm{b}}+\beta k_{\mathrm{s}}[\mathrm{He}]}
$$

The large increase in $k_{2 \mathrm{nd}}^{\mathrm{abs}}$ between $395 \mathrm{~K}$ and $155 \mathrm{~K}$ is due to a corresponding increase in complex lifetime (decrease in $k_{\mathrm{b}}$ ). We are modeling these changes, as well as those due to changing helium pressure, and substituting $\mathrm{CD}_{4}$ for $\mathrm{CH}_{4}$ using a phase space model. The results will be reported later [43].

\section{Conclusions}

1. A new instrument has been described that is designed to study thermal energy reaction rates and product distributions of mass-selected ions. The first stage of the instrument, which generates and mass selects the ions of interest, is a reversegeometry double-focusing mass spectrometer.

2. The new portion of the instrument, following the first-stage mass spectrometer, consists of deceleration and focusing lenses that retard the $5-\mathrm{keV}$ ions and focus them into a high-pressure gas cell (pressure $=1-2$ torr $\mathrm{He}$ ). Ions are drifted through the cell by a small electric field. Reactions occur with trace amounts of neutral reactant $\left(10^{-5}-10^{-2}\right.$ torr). Products that exit the cell are analyzed with a quadrupole mass filter.

3. Important aspects of ion energy, ion residence time, and ion thermalization are discussed in detail.

4. Applications of the instrument include bimolecular and clustering reactions of unusual species (cluster ions and electronically excited ions) and ion mobilities. These processes can also be studied as a function of temperature from 100 to $600 \mathrm{~K}$. Bimolecular rate constants can be measured from the collision limit near $1 \times 10^{-9} \mathrm{~cm}^{3} / \mathrm{s}$ to as low as $\sim 1 \times 10^{-14}$ $\mathrm{cm}^{3} / \mathrm{s}$.

\section{Acknowledgment}

The support of the National Science Foundation under grant CHE88-17201 is gratefully acknowledged.

\section{References}

1. (a) Adams, N. G.; Smith, D. Int. J. Mass Spectrom, Ion Processes 1976, 21, 349. (b) Smith, D.; Adams, N. G. In Gas Phase Ion Chemistry; Bowers, M. T., Ed.; Academic; New York, 1979; Vol. 1, p 1 .

2. McFarland, M.; Albritton, D. L.; Fehsenfeld, F. C.; Ferguson, E. E.; Schmeltekopf, A. L. J. Chem. Phys. 1976, 59, 6610.

3. Van Doren, J. M.; Barlow, S. E.; DePuy, C. H.; Bierbaum, V. M. Int. I. Mass Spectrom. Ion Processes 1987, 81, 85.

4. (a) Smith, D. L.; Futrell, J. H. Int. J. Mass Spectrom. Ion Processes 1974, 14, 171. (b) Kemper, P. R.; Bowers, M. T. Int. J. Mass Spectrom. Ion Processes 1983, 52, 1.

5. Armentrout, P. B.; Beauchamp, J. L. I. Chem. Phys. 1981, 74, 2819.

6. Ervin, K. M.; Armentrout, P. B. J. Chem. Phys. 1985, 83, 166.

7. (a) Lebrilla, C. B.; Amster, I. J.; Mclver, R. T. Int. J. Mass Spectrom. Ion Processes 1989, 87, R7. (b) Mclver, R. T.; Hunter, R. L.; Bowers, W. D. Int. J. Mass Spectrom. Ion Processes 1985, 64,67 .
8. Kofel, P.; Alleman, M.; Kellerhals, H.; Wanczek, K. P. Int. J Mass Spectrom. Ion Processes 1986, 72, 53; 1989, 87, 237.

9. Shukla, A. K.; Anderson, S. G.; Howard, S. L.; Sohlberg, K. W.; Futrell, J. H. Int. J. Mass Spectrom. Ion Processes 1988, 86,61 .

10. Bohringer, H.; Arnold, F. J. Chem. Phys. 1982, 77, 5534.

11. Bohringer, H.; Arnold, F. Int. J. Mass Spectrom. Ion Processes $1983,49,61$.

12. Jarrold, M. F,; Bower, J. E.; Creegan, K. J. Chem. Phys. 1989, $90,3615$.

13. Morgan, R. P.; Beynon, J. H.; Bateman, R. H.; Green, B. N. Int. J. Mass Spectrom. Ion Processes 1978, 28, 171.

14. Dushman, S. In Scientific Foundation of Vacuum Technique; Lafferty, J. M., Ed.; Wiley: New York, 1962.

15. Holland, L.; Steckelmacher, W.; Yarword, J. Vacuum Manual; Halstead Press/Wiley: New York, 1974 (note typographical errors).

16. Shumacher, B. W. Trans. 8th Int. Vac. Symp. 1961, 2, 1192.

17. Poulter, K. F.; Rodgers, M.-J.; Thompson, T. J.; Perkin, M. P. Vacuum 1983, 33, 311.

18. McDaniel, E. W.; Mason, E. A. The Mobility and Diffusion of Ions in Gases; Wiley: New York, 1973.

19. Chang, C.; Sroka, G. J.; Meisels, G. G. Int. J. Mass Spectrom. Ion Processes 1973, 11, 367.

20. Albritton, D. L. In Kinetics of Ion-Molecule Reactions; Ausloos, P., Ed.; Plenum: New York, 1979.

21. Viehland, L. A.; Lin, S. L.; Mason, E. A. Chemt. Phys. 1981, 54,341 .

22. Lindinger, W. Int. J. Mass Spectrom. Ion Processes 1987, 80, 115.

23. Lindinger, W.; Howorka, F.; Lukac, P.; Kuhn, S.; Villinger, H.; Alge, E.; Ramler, H. Phys. Rev. A 1981, 23, 2319.

24. Vestal, M. L.; Blakley, C. R.; Ryan, P. W.; Futrell, J. H. Rev. Sci. Instrum. 1976, 47, 15.

25. Futrell, J. H.; Miller, C. D. Rev. Sci. Instrum. 1966, 37, 1521.

26. Heddle, D. W. O. J. Phys. E 1971, 4, 981.

27. (a) Wannier, G. H. Phys. Rev, 1951, 83, 281. (b) Wannier, G. H. Bell Syst. Tech. J. 1953, 32, 170.

28. Albritton, D. L.; Dotan, I.; Lindinger, W.; McFarland, M.; Tellinghuisen, J.; Fehsenfeld, F. C. J. Chem. Phys. 1977, 66, 410.

29. Smith, D.; Adams, N. G. Phys. Rev. A 1981, 23, 2327.

30. Lindinger, W.; Albritton, D. L. J. Chem. Phys. 1975, 62, 3517.

31. Ellis, H. W.; Pai, R. Y.; McDaniel, E. W.; Mason, E. A.; Viehland, L. A. Atomic Data Nucl. Data Tables 1976, 17, 177; 1978, 22, 179; 1984, 31, 113.

32. Twiddy, N. D.; Mohebati, A.; Tichy, M. Int. J. Mass Spectrom. Ion Processes 1986, 74, 251.

33. Rowe, B. R.; Fahey, D. W.; Fehsenfeld, F. C.; Albritton, D. L. J. Chem. Phys. 1980, 73, 194.

34. Helm, H.; Elford, M. T. J. Phys. B 1978, 11, 3939.

35. Kemper, P. R.; Bowers, M. T. J. Am. Chem. Soc. (in press).

36. Stori, H.; Alge, E.; Villinger, H.; Egger, F.; Lindinger, W. Int. J. Mass Spectrom. Ion Processes 1979, 30, 263.

37. Bolden, R. C.; Hemworth, R. S.; Shaw, M. J.; Twiddy, N. D. J. Phys, B 1970, 3, 45 .

38. Shul, R. J.; Upschulte, B. L.; Passarella, R.; Keesee, R. G.; Castleman, A. W., Jr. J. Phys. Chem. 1987, 91, 2556.

39. Adams, N. G.; Bohme, D. K.; Dunkin, D. B.; Fehsenfeld, F. C. J. Chem. Phys. 1970, 52, 1951.

40. Adams, N. G.; Smith, D. Int. J. Mass Spectrom. Ion Processes $1976,21,349$.

41. Dotan, I.; Lindinger, W. J. Chem. Phys. 1982, 76, 4972.

42. Raksit, A. B.; Stock, H. M. P.; Waveing, D. P.; Twiddy, N. D. J. Phys. B 1978, 11, 4237.

43. Kemper, P. R.; van Koppen, P. A. M.; Bowers, M. T., to be published.

44. Tonkyn, R.; Ronan, M.; Weisshaar, J. J. Phys. Chem. 1988, 92, 92. 